\title{
Definir un entorno virtual para la enseñanza y aprendizaje (EPA)', criterios y enseñanzas
}

\section{Define a virtual environment for teaching and learning (EPA), criteria and teachings}

Recibido: 8 de abril de 2020 / Aprobado: 15 de mayo de 2020

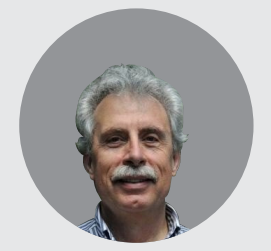

DR. JOAN RUÉ DOMINGO

UNIVERSITAT AUTÒNOMA DE BARCELONA joan.rued@gmail.com

\section{Resumen}

La vigente cultura docente, orientada fundamentalmente desde la lógica de la comunicación vertical, la estandarización y la evaluación externa debe reconfigurarse para adaptarse a las necesidades y características del trabajo no presencial de los estudiantes, mediante intercambios virtuales, basados en la comunicación horizontal y en red, en la personalización de los mensajes, así como en la autorregulación y autoevaluación. Este texto describe y argumenta el proceso de creación, aplicación y desarrollo de las tecnologías virtuales a las relaciones de enseñanza-aprendizaje en la universidad, de acuerdo con el concepto de Entorno de Aprendizaje

Personalizado-Portafolio (EPA) que designa la propuesta elaborada y analizada aquí que se halla en concordancia con la propuesta de EDUCAUSE, (Brown, Dehoney y Millichap, 2015). No se trata de emplear herramientas sueltas, sino integrarlas en una sola plataforma, con sus diversas funcionalidades. El modelo EPA desarrollado se configuró desde la pretensión de ir más allá del empleo de una variedad de herramientas virtuales. La reflexión desarrollada acerca del empleo de estos recursos por parte de los diversos profesores y estudiantes, y a partir de una amplia encuesta y recogida de valoraciones de los estudiantes, puso de manifiesto, entre otros aspectos importantes, que cualquier tentativa del profesorado de desarrollar e implementar en las propias clases un determinado EPA requiere, por su parte, la asunción de un rol que se podría denominar como de 'agente del cambio' en lugar del de consumidor pasivo de plataformas digitales cuyos diseños le son ajenos. Dicha capacidad de agencia de cambio, no obstante, requiere tomar en cuenta algunos aspectos que se consideran al final.

Palabras clave: Enseñanza universitaria; plataformas digitales; aprendizaje; reflexión; autoevaluación; personalización

\section{Abstract}

The current teaching culture, mainly oriented from the trends of the vertical communication, standardization and external evaluation, must be reconfigured to adapt to the needs and

1. Este artículo es una reelaboración de la tercera parte de: Rué, J. (2015). Entornos de aprendizaje digitales y calidad en la Enseñanza Superior, Barcelona, UOC. Disponible en http://www.editorialuoc.cat/entornos-de-aprendizaje-digitales-y-calidad-en-la-educacion-superior

a. Pedagogo, profesor de la Unidersitat Autònoma de Barcelona UAB, retirado. Para contactar al autor: joan.rued@gmail.com 
characteristics of the remote learning, through virtual exchanges. Opposite to the former, these are based on both, horizontal communication and on network, besides the personalization of messages, as well as in self-regulation and self-evaluation.

This text describes and argues in favour of the process of creation, application and development of virtual technologies that support the teaching-learning relationship in the university. The model depicted here is framed in the concept of Personalized Learning Environment-Portfolio (EPA) that designates the analyzed proposal. Its conceptual support is consistent with the EDUCAUSE proposal (Brown, Dehoney and Millichap, 2015), that is, the rejection of using individual tools, but integrating all of them into a single platform, with its various functionalities.

The developed EPA model was configured according to the purpose of going beyond the use of a variety of virtual tools. The reflection developed about using these resources by teachers and students is based on data gathered from a wide survey and collection of student evaluations. Among other important aspects, these data revealed that any attempt by teachers to develop and implement a certain EPA in their courses, requires the assumption of a role that could be termed as 'agent of change' instead of that of passive consumer of digital platforms whose designs are alien to a specific teaching. This capacity of adopting the role of changing agency, however, requires taking into account some aspects that are considered in the conclusions.

Keywords: Higher education teaching; digital platforms; learning; reflection; selfevaluation; personalization

\section{Introducción}

La súbita aparición del Covid 19 en el mundo, además de dolor, enormes pérdidas económicas y graves problemas sociales, ha puesto de manifiesto, no obstante, el potencial de los intercambios virtuales en el trabajo así como en la enseñanza. En este caso también se ha reconsiderado su potencial formativo, en muchos países, debido a la imposibilidad de seguir con normalidad con la enseñanza presencial.

La experiencia relativamente reciente en este campo ha evidenciado, sin embargo, que el empleo de recursos digitales en las relaciones de enseñanza y aprendizaje ( $r E-A)$ es una realidad que hay que aprender a manejar de forma adecuada.

La primera consideración es de orden político y apunta a interrogar sobre las oportunidades que se brindan desde las instituciones y quiénes son los beneficiarios de dichas oportunidades. No hay que ignorar que muchos ciudadanos y familias, incluso de universitarios, no tienen acceso todavía a las máquinas o a internet, por lo que la denominada alfabetización digital tan solo lo es de las personas pertenecientes a los sectores más acomodados de la sociedad. Este es un dato importante a considerar cuando desde las instituciones se aboga por la enseñanza virtual.

La segunda consideración es de orden pedagógico. En efecto, si exceptuamos las enseñanzas manifiestamente on line y para públicos específicos, la introducción de la virtualidad en la formación la contemplamos como un excelente complemento de las relaciones directas en el aula y con un alto valor estratégico. No obstante, en todos los casos, su introducción y forma de manejo debe ceñirse a la lógica pedagógica antes que a la tecnológica cuando se aspira a ejercer una verdadera eficacia formativa con las herramientas virtuales y no emplearlas 
tan solo como un producto comercial considerado como "moderno" o de "prestigio".

De acuerdo con estas consideraciones, las herramientas deberán supeditarse a las intencionalidades y características pedagógicas de la relación de enseñanza y aprendizaje mediante entornos virtuales. Ello presupone que la vigente cultura docente, orientada fundamentalmente desde la lógica de la comunicación vertical, la estandarización y la evaluación externa, deberá ajustarse a las necesidades y características del trabajo no presencial, mediante intercambios virtuales, basados fundamentalmente en la comunicación horizontal y en red, en la personalización de los mensajes y en la autorregulación y autoevaluación. Pero más importante aún, es no olvidar tampoco la cultura mediante la cual se están socializando los jóvenes con respecto a las tecnologías, caracterizada por la frecuencia de su empleo, las relaciones mediante redes, 0 la de la inmediatez de los feed-back y la personalización de los mensajes recibidos.

En cualquier caso, la relación educativa mediante plataformas deberá llevarse a cabo sin descuidar su principal propósito, el de la calidad de los aprendizajes.

Se puede afirmar, en consecuencia, que ninguna tecnología aplicada a la enseñanza y aprendizaje virtuales será verdaderamente eficiente sin tres grandes condiciones: un propósito pedagógico claro, un modelo-marco relevante para el empleo de las diversas herramientas tecnológicas y un uso eficiente de las mismas, a fin de potenciar la autonomía de todos los estudiantes y la calidad de sus aprendizajes. La principal razón de ello es que el aprendizaje mediante recursos virtuales no consiste "en otra manera de enseñar", sino en "otra manera de aprender".

Veamos lo que mostraba un estudio relativamente reciente (EIC, 2018), comparando la percepción de docentes de varios países latinoamericanos y España con respecto a la introducción de las tecnologías virtuales en la enseñanza. Entre otros aspectos, se constataba lo siguiente, considerando las respuestas agregadas de todos los docentes:

Se valoran las tecnologías porque se cree que permiten acceder a un mayor número de contenidos, facilitan que los estudiantes aprendan a comunicarse mediante diversos lenguajes y medios de expresión, o que les facilitan una potencial adaptación a su futuro laboral. Asimismo, más de la mitad de los docentes en todos los países creen que el uso de las tecnologías potencia el aprendizaje autónomo.

Sin embargo, tan solo la mitad o menos de los docentes encuestados estaban de acuerdo con que el aprendizaje debía estar adaptado a las necesidades de los alumnos, que estaba potenciando su capacidad crítica, que era útil para contrastar fuentes informativas y que permitía una comunicación más fluida entre docentes y estudiantes.

Ambas valoraciones manifiestan creencias contradictorias. Los argumentos para la valoración positiva de las tecnologías no apuntan hacia una clara percepción de que un aprendizaje autónomo requiere precisamente de los aspectos que el profesorado consideraba poco o relativamente poco desarrollados, es decir, los argumentados con respecto a la capacidad crítica, el contraste de fuentes, etc.

En las líneas siguientes se describe y argumenta a favor del proceso de creación, aplicación y desarrollo de las tecnologías virtuales para sostener la enseñanza y el aprendizaje en la universidad y como apoyo a las asignaturas de carácter presencial. La elaboración del modelo descrito aquí abarcó desde el curso 2009-2010 hasta el 2012-2013, en un proceso complejo de investigación en la acción, llevado a cabo por diez docentes universitarios en tres universidades y nueve titulaciones. Contó con la recogida de valoraciones de los 
estudiantes mediante encuestas cerradas y aportaciones abiertas, a partir de sucesivos ensayos prácticos.

\section{Emprender la experiencia de generar una plataforma de aprendizaje en docencia universitaria. Sus puntos de partida}

Bhattacharya (2001), Biggs (2003), Cockburn, Carver, Shirley y Davies (2007), entre otros, inspiraron nuestro trabajo en la Universitat Autònoma de Barcelona (UAB) sobre la calidad del aprendizaje mediante recursos virtuales, que englobamos en to que denominamos como Entorno Personal para el Aprendizaje-Portafolio del estudiante (EPA) (Rué et al. 2010). Es decir, no se trataba de emplear herramientas sueltas, sino integrarlas en una sola plataforma, con sus diversas funcionalidades. Todo ello lo concretamos empotrando la herramienta Mahara, de código abierto, que permite el diseño de un interface personalizado para la materia y para el estudiante, dentro de Moodle, dado que son perfectamente compatibles. El concepto de Entorno de Aprendizaje Personalizado-Portafolio que designa la propuesta elaborada y analizada aquí, se define en concordancia con la propuesta de EDUCAUSE (Brown, Dehoney y Millichap, 2015, p.10): "un conjunto de aplicaciones y de plataformas que se proporcionan a los alumnos mediante un portafolio que cuenta con un conjunto integrado de herramientas (digitales)".

Brown et al. (2015) exploraron las diferencias o la distancia entre las herramientas de gestión de aprendizaje actuales y el tipo de entorno de aprendizaje digital de nueva generación que pudiera satisfacer las necesidades cambiantes de la educación superior. A partir de una extensa consulta a más de 70 líderes de opinión en el ámbito de estos recursos, concluyeron que los aspec- tos imprescindibles de esta herramienta de nueva generación serían: la interoperabilidad; la personalización; su funcionalidad para el análisis; el asesoramiento y evaluación del aprendizaje; la colaboración; y la accesibilidad a la misma, gracias al carácter universal de su diseño.

Dado que ninguna aplicación por sí misma puede ofrecer todos esos dominios, tal como concluye dicho informe, se recomienda un enfoque de "Lego" para el desarrollo de este entorno digital de nueva generación para el aprendizaje. Es decir, los diversos componentes de este nuevo medio deberán poder estar acoplados de modo que permitan a los individuos e instituciones la oportunidad de construirse entornos adaptados a sus necesidades y objetivos de aprendizaje. Según los autores del informe, su arquitectura sería una confederación de sistemas TIC. En la tabla 1 se describen sucintamente dichas características y lo que significan.

Se observará que en su configuración, se destaca el valor de la contextualización y de la personalización. En otras palabras, no serán exactamente lo mismo para los estudiantes, para los docentes y para cualquier institución. Se asume que, desde el punto de vista de los usuarios, será como un espacio en la 'nube' que permita agregar y conectar contenido y funcionalidades, donde los mismos usuarios realizarán su propia adaptación y ajuste de sus entornos, a partir de aplicaciones auto-seleccionadas y descargables. Su modelo de arquitectura será el de la heterogeneidad para producir homogeneidad mediante una fusión de elementos dispares. El resultado de este enfoque es una aplicación que usa contenidos provenientes de más de una fuente para crear un nuevo servicio el cual se muestra a través de una sola interfaz gráfica. 
Tabla 1. Aspectos imprescindibles de las herramientas EPA/Portafolio de nueva generación

\begin{tabular}{|c|c|}
\hline ASPECTO & RASGOS QUE LO DEFINEN \\
\hline Interoperabilidad & $\begin{array}{c}\text { Todos los componentes deben ser capaces de aceptar y de intercambiar contenidos curriculares en } \\
\text { formatos comunes, para permitir los intercambios, las transferencias y su uso. 2- Las diversas herramientas } \\
\text { deben poder ser incorporadas al entorno, de modo autónomo y fácilmente por los usuarios finales. 3- El } \\
\text { ambiente de aprendizaje será la principal fuente de datos para el aprendizaje. El intercambio sin trabas de } \\
\text { los datos es imprescindible para poder sumar, integrar y analizar los datos de aprendizaje. 4- la plataforma } \\
\text { debe permitir la creación de nuevos estándares de interoperabilidad de manera que se dé una coherencia } \\
\text { global entre todos sus componentes. }\end{array}$ \\
\hline Personalización & $\begin{array}{c}\text { Es el factor tangible más importante para dar forma a la experiencia del usuario. Un ecosistema de } \\
\text { aprendizaje que permite a los alumnos y docentes actuar como arquitectos de su propio entorno es una } \\
\text { herramienta de gran alcance. La arquitectura personalizada remite al usuario final, pero también a los } \\
\text { departamentos, materias e instituciones. Es decir, requiere un enfoque de colectivo por encima del } \\
\text { personal. También remite a la posibilidad de recibir apoyos específicos para las necesidades de } \\
\text { aprendizaje de cada usuario. }\end{array}$ \\
\hline $\begin{array}{l}\text { Funcionalidad para el análisis; } \\
\text { el asesoramiento y evaluación } \\
\text { del aprendizaje }\end{array}$ & $\begin{array}{l}\text { Remite al tipo de datos para dicho análisis: personales y grupales; disposición, actitud y seguimiento de la } \\
\text { materia; a los artefactos y contenidos generados por cada usuario. También al análisis de los aprendizajes } \\
\text { para todos los interesados. Debe orientarse, por lo menos, hacia tres propósitos: el empoderamiento de los } \\
\text { estudiantes, la mejora continuada de la enseñanza continua y la supervisión institucional. }\end{array}$ \\
\hline Colaboración & $\begin{array}{l}\text { El apoyo a la colaboración debe ser un objetivo principal en el diseño. Las materias desarrolladas en } \\
\text { entornos sociales auténticos propician procesos de aprendizaje de gran valor. Para ello, los portafolios y } \\
\text { herramientas para la creación de contenidos deben integrarse plenamente en este medio. }\end{array}$ \\
\hline $\begin{array}{c}\text { Accesibilidad en su diseño } \\
\text { universal }\end{array}$ & $\begin{array}{l}\text { Considera al usuario como receptor y creador de contenidos. Su diseño universal tiene el potencial de } \\
\text { proporcionar el entorno de aprendizaje digital más accesible posible. Por 'diseño universal' se entiende 'el } \\
\text { diseño de productos y entornos que puedan utilizar todas las personas, en la mayor medida posible, sin } \\
\text { necesidad de adaptación ni diseño especializado. }\end{array}$ \\
\hline
\end{tabular}

Fuente: EDUCAUSE, Brown et al., 2015.

Atendiendo a lo anterior, el modelo EPA que desarrollamos se configuró a partir de cuatro propósitos básicos, con la pretensión de ir más allá del empleo de una variedad de herramientas virtuales. En efecto, se consideró que este modelo debería:

- Constituir un entorno de aprendizaje personal virtual, funcional para ser implementado en el desarrollo de los aprendizajes en los diferentes grupos, en las diversas asignaturas e instituciones universitarias. Dicho entorno debía ejercer las funciones de portafolio.

- Visualizarse como un entorno virtual-herramienta que facilitara el hecho de aprender, desde un andamiaje fiable para los estudiantes.

- Facilitar la autonomía de los alumnos y su capacidad de agencia en la auto-conducción de su aprendizaje, en las distintas materias. 
- Favorecer la calidad de los aprendizajes en los diversos entornos institucionales (asignaturas y grupos), independientemente del tamaño del grupo de estudiantes.

\section{Las decisiones adoptadas por la red de docentes implicados en la experiencia}

En relación con el propósito pedagógico (el para qué) nos plateamos unas preguntas iniciales que fueron configurando el diseño de los EPA en cada materia coordinada en el proyecto. Eran las siguientes:

a En qué medida se les facilitará a los estudiantes la oportunidad de:

- Reflexionar sobre todas sus actividades y logros y evaluarlos.

- Potenciar un pensamiento reflexivo.

- Reflexionar sobre el desarrollo continuado y la profundidad de su conocimiento y competencias.

- Incrementar la confianza en ellos mismos y ayudarles a identificar aquellas habilidades que requieren ser mejoradas.

b Qué rasgos básicos debería recoger un portafolio que incorpore elementos de la web con el fin de:

- Mejorar los aprendizajes individuales.

- Facilitar la colaboración y cooperación entre los estudiantes en la generación de dichos aprendizajes.

- Aportar una mayor autonomía y capacidad de auto-regulación.

La respuesta a dichas cuestiones suponía una selección de los medios a ser empleados y su integración o interconexión en sus funcionalidades, pues no todos los recursos tecnológicos empotrados en una plataforma son susceptibles de ser empleados simultáneamente 0 funcionalmente con un grupo grande de estudiantes.

\section{Los rasgos distintivos del modelo propuesto}

En relación con el propósito pedagógico (el para qué) nos plateamos unas preguntas iniciales que fueron configurando el diseño de los EPA en cada materia coordinada en el proyecto. Eran las siguientes:

- Facilitar la confrontación de los estudiantes con algunos retos relevantes para su aprendizaje (p.e. ejercicios, casos, problemas, análisis de hechos, de documentos, elaboración de síntesis, de puntos de vista argumentados, de selección de evidencias, etc.).

- Facilitar la posibilidad de interconexión de las propias ideas con las de otros, en una elaboración compleja de las respuestas.

- Poder incorporar y almacenar la información indispensable para el desarrollo de aquella confrontación, suministrada por el docente, por el propio estudiante o los iguales.

- Adoptar un formato de portafolio capaz también de mostrar que el error es posible y que lo relevante, en la evaluación de los aprendizajes, es todo el proceso de resolución, seguido para la superación de los retos propuestos.

En este sentido, era muy importante que el empleo de las diversas herramientas seleccionadas permitiera las funcionalidades de comunicación y de control de los tiempos para estimular un proceso de aprendizaje continuado y sostenido en el tiempo, dada la importancia de este aspecto en el desarrollo de aprendizajes de calidad. 
También debía integrar las herramientas para trabajar de modo eficaz, tanto individualmente como en colaboración con los demás para que los estudiantes pudieran auto-regular su aprendizaje y reflexionar sobre su propio trabajo, unas condiciones necesarias para generar un aprendizaje profundo.

Además de lo anterior, era necesario que incorporara funcionalidades para el registro de los progresos de los estudiantes y la posibilidad de discutirlos y evaluar su progreso. Es decir, funcionalidades de registro de las evidencias que atestiguaran tanto la cantidad de recursos empleados en la resolución del trabajo como de sus sucesivos progresos. Cabe hacer notar que esta condición tiende, por sí misma, a ser un elemento muy importante para el control del plagio en los trabajos. Dichos requerimientos se ilustran en la Figura 1.

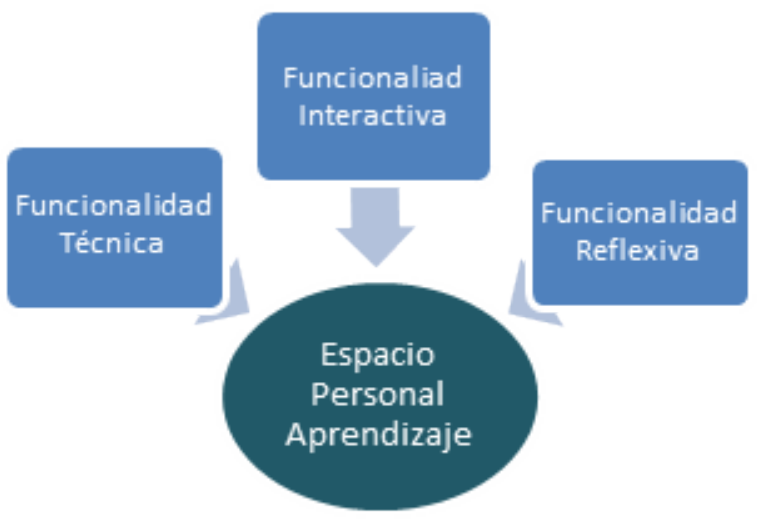

Figura 1. Funcionalidades clave en la configuración de un EPA.

La tabla 2 describe la arquitectura pedagógica del modelo desarrollado. Detalla los diez rasgos que se seleccionaron para definir las tres grandes funcionalidades básicas apuntadas. Dichos rasgos fueron seleccionados y consensuados en equipo después de una reflexión basada en las observaciones de las conductas de estudiantes y profesores, así como mediante procesos de encuesta, a lo largo de las experiencias piloto llevadas a cabo entre 2009 y 2011.
Tabla 2. Las funcionalidades esperadas para un EPA portafolio y sus rasgos distintivos

\begin{tabular}{|c|c|}
\hline FUNCIONALIDADES & RASGOS QUE LAS DEFINEN \\
\hline \multirow{5}{*}{ Técnica } & $\begin{array}{c}\text { Permite resolver las actividades de } \\
\text { estudiantes y profesores con la máxima } \\
\text { funcionalidad posible. }\end{array}$ \\
\hline & $\begin{array}{l}\text { Se combinan o integran funcionalmente } \\
\text { diversos recursos multimedia, disponibles } \\
\text { en la web. }\end{array}$ \\
\hline & $\begin{array}{c}\text { Facilita la recuperación, por parte de los } \\
\text { usuarios, de la información recogida en la } \\
\text { plataforma, garantizando la seguridad y } \\
\text { privacidad del espacio y la información } \\
\text { acumulada. }\end{array}$ \\
\hline & $\begin{array}{c}\text { Aporta una capacidad de memoria de } \\
\text { almacenamiento suficiente. }\end{array}$ \\
\hline & $\begin{array}{l}\text { Permite incorporar recursos de recopilación } \\
\text { y de archivo ordenado de materiales y } \\
\text { evidencias, individuales y de equipo. }\end{array}$ \\
\hline \multirow{2}{*}{$\begin{array}{l}\text { Interactiva y } \\
\text { colaborativa del } \\
\text { estudiante }\end{array}$} & $\begin{array}{c}\text { Posibilita trabajar individualmente, } \\
\text { compartir el trabajo con otros y colaborar } \\
\text { en el mismo. }\end{array}$ \\
\hline & $\begin{array}{l}\text { Permite incorporar recursos facilitados por } \\
\text { los docentes y por los propios estudiantes } \\
\text { que promueven su autonomía personal y en } \\
\text { grupo en el trabajo (documentación, } \\
\text { orientaciones para actuar, calendario, } \\
\text { herramientas facilitadoras del trabajo, etc.) }\end{array}$ \\
\hline \multirow{3}{*}{$\begin{array}{l}\text { (Co)Reflexiva y } \\
\text { (co)Reguladora }\end{array}$} & $\begin{array}{l}\text { Facilita un recurso y espacio explícitos para } \\
\text { las reflexiones del estudiante. }\end{array}$ \\
\hline & $\begin{array}{c}\text { Permite al estudiante, a sus iguales y al } \\
\text { docente el seguimiento funcional, y con } \\
\text { economía de tiempo, de los trabajos } \\
\text { individuales. }\end{array}$ \\
\hline & $\begin{array}{c}\text { Facilita la interacción fluida con el } \\
\text { profesorado /con los iguales/ para recibir el } \\
\text { necesario feed-back respecto de las } \\
\text { actividades emprendidas por el estudiante } \\
\text { y entre los estudiantes colaboradores en los } \\
\text { mismos aprendizajes. }\end{array}$ \\
\hline
\end{tabular}

Fuente: Rué (2015) 
A su vez, la Tabla 3, describe el modo mediante el cual, a partir de los recursos disponibles en cada caso, los diversos profesores y profesoras participantes de esta experiencia de reflexión y acción han ido configurando su EPA/Portafolio virtual. En ella se relacionan los recursos realmente utilizados en la interacción con los estudiantes, de acuerdo con las posibilidades de cada tipo de plataforma institucional de la que podían disponer y los propósitos de cada profesor y para algunos de los propósitos clave en toda relación de enseñanza y aprendizaje, tanto desde el punto de vista del docente como del estudiante:

¿Qué debe o deberá hacer/ qué debo o deberé hacer?
¿Cómo persistirá en el trabajo propuesto /hasta dónde me involucro en el trabajo?

¿Qué han hecho, qué resultados obtienen/ qué he realizado, cómo lo hice?

Las herramientas mencionadas aquí son las que estaban disponibles en 2015. Sin embargo, a pesar de que ilustran perfectamente los argumentos que se aportan, estas herramientas no están actualizadas y no reflejan aquellas actualmente disponibles, mucho más variadas y con nuevas funcionalidades, como pueden ser la plataforma Zoom, las incorporadas en Google para el trabajo y la relación en equipos u otras.

¿Cómo lo hará/ Cómo lo haré?

Tabla 3. Propósitos y herramientas que definen un EPA

\begin{tabular}{|c|c|}
\hline $\begin{array}{c}\text { PROPÓSITO: APORTAR INFORMACIÓN BÁSICA NECESARIA PARA GUIAR AL } \\
\text { ESTUDIANTE (¿QUÉ DEBO HACER?) }\end{array}$ & HERRAMIENTAS DIGITALES \\
\hline $\begin{array}{l}\text { Facilitar acceder a la información cuando y desde donde desee el estudiante. } \\
\text { Informar sobre la Guía docente; Bibliografía básica; Orientaciones generales del curso; } \\
\text { Determinadas temáticas, presentaciones y materiales escritos. próximos eventos, anuncios, } \\
\text { Informar del cronograma, hacerse una agenda. Conocer anticipadamente las fechas de teoría, } \\
\text { de clases prácticas y de exámenes. } \\
\text { Publicar notas, enlaces de interés y videos. } \\
\text { Informar sobre próximos eventos, anuncios, publicación de notas, enlaces de interés. }\end{array}$ & \multirow{2}{*}{$\begin{array}{l}\text { Carpetas, ficheros temáticos: docs, } \\
\text { pdf y ptt. } \\
\text { YouTube } \\
\text { Webs seleccionadas } \\
\text { Calendario } \\
\text { Moodle } \\
\\
\text { Mensajes de correo, } \\
\text { WhatsApp } \\
\text { Foros }\end{array}$} \\
\hline $\begin{array}{l}\text { Aportar ejercicios, tareas, apuntes y presentaciones. } \\
\text { Aclarar puntos débiles en la comprensión de los estudiantes. } \\
\text { Presentar, videos, ppt, ejercicios y tareas, apuntes y presentaciones. } \\
\text { Enviar online trabajos prácticos y tareas. }\end{array}$ & \\
\hline $\begin{array}{l}\text { PROPÓSITO: AYUDAR A ORGANIZAR LA ACCIÓN DEL ESTUDIANTE EN SU } \\
\text { TRABAJO (¿CÓMO LO HAGO?) }\end{array}$ & HERRAMIENTAS DIGITALES \\
\hline $\begin{array}{l}\text { Aportar orientaciones específicas sobre tareas determinadas; objetivos; resultados esperados; } \\
\text { orientaciones sobre la estructura del portafolio. } \\
\qquad \text { Facilitar el trabajo autónomo del estudiante. } \\
\text { Proponer el desarrollo de actividades, descarga de ejercicios y actividades, visionado de videos. } \\
\qquad \text { Facilitar la auto-corrección. } \\
\text { Facilitar realizar actividades en grupo (evaluativas o no evaluativas). }\end{array}$ & $\begin{array}{l}\text { Carpeta Digital, Moodle } \\
\text { Guías de trabajo para cada tema. } \\
\text { Foros de discusión y debate. } \\
\text { Correo electrónico. } \\
\text { YouTube. } \\
\text { Propuestas de actividades. } \\
\text { Videos: contenidos explicados y } \\
\text { trabajados en clase. }\end{array}$ \\
\hline
\end{tabular}




\begin{tabular}{|c|c|}
\hline $\begin{array}{l}\text { PROPÓSITO: AYUDAR A SOSTENER Y ANIMAR LA ACCIÓN DEL ESTUDIANTE } \\
\text { EN SU TRABAJO O ESTUDIO (CÓMO SOSTENER EL INTERÉS MÁS ALLÁ DE LO } \\
\text { INDISPENSABLE) }\end{array}$ & HERRAMIENTAS \\
\hline 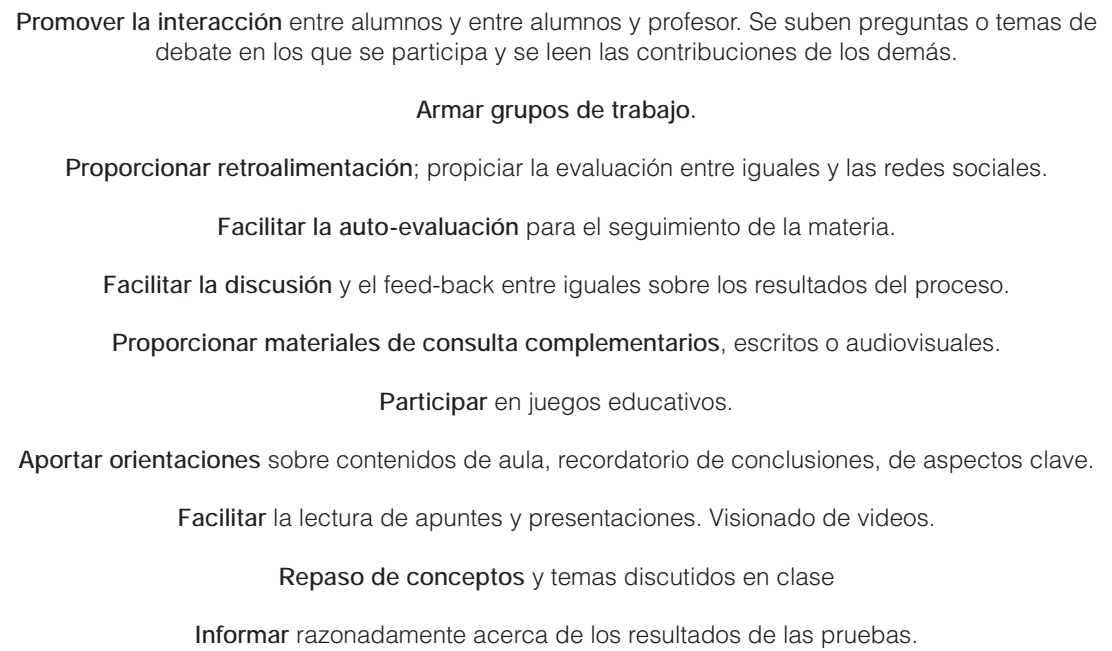 & $\begin{array}{c}\text { Carpeta Digital, Moodle, } \\
\text { Correo electrónico } \\
\text { Taller (Moodle) } \\
\text { Fórum } \\
\text { Correo electrónico } \\
\text { Rúbricas } \\
\text { WhatsApp } \\
\text { Webs específicas } \\
\text { Programas de simulación en red } \\
\text { Vídeos específicos, } \\
\text { YouTube, } \\
\text { Wikipedia }\end{array}$ \\
\hline $\begin{array}{l}\text { PROPÓSITO: AYUDAR A LOS ESTUDIANTES A REFLEXIONAR, AUTORREGULARSE Y } \\
\text { AUTOEVALUARSE (¿QUÉ ESTOY HACIENDO, QUÉ RESULTADOS, CÓMO LO VALORO?) }\end{array}$ & HERRAMIENTAS \\
\hline $\begin{array}{l}\text { Informar sobre el estado del portafolio; sobre tareas concretas; propuestas concretas. } \\
\text { Responder a preguntas/dudas/consultas. } \\
\text { Aportar comentarios sobre conceptos, pasos, realizados. } \\
\text { Dar feed-back sobre algún trabajo práctico o examen; sobre las cuestiones } \\
\text { que los estudiantes plantean. } \\
\text { Promover la reflexión: llevar diarios reflexivos, ofrecer, proponer criterios de evaluación, } \\
\text { grabaciones de ciertas actividades (simulaciones), agenda, calendario. } \\
\text { Fomentar el aprendizaje en autonomía y cooperación, para mejorar la reflexión y } \\
\text { el razonamiento propio. } \\
\text { Facilitar la evaluación del propio aprendizaje. } \\
\text { Sintetizar los aprendizajes desarrollados por los estudiantes en las sucesivas sesiones de trabajo. } \\
\text { Informar. Listados de notas de prácticas, parciales y finales. } \\
\text { Auto-evaluación del portafolio de cada estudiante mediante rúbrica. } \\
\text { Dar feed-back mediante devoluciones individuales y colectivas. } \\
\text { Facilitar la auto-evaluación para el seguimiento de la materia. Revisión mutua de los } \\
\text { trabajos entre estudiantes de un pequeño grupo. } \\
\text { Argumentar las razones de las notas dadas y criterios de corrección. }\end{array}$ & $\begin{array}{c}\text { Carpeta Digital, Moodle, } \\
\text { YouTube } \\
\text { Webquest o similar } \\
\text { Correo electrónico } \\
\text { Vídeos sobre los aprendizajes realizados } \\
\text { Cuestionarios moodle } \\
\text { Blog } \\
\text { Carpeta del estudiante } \\
\text { Rúbricas }\end{array}$ \\
\hline
\end{tabular}

Fuente: Rué (2015)

A partir del desarrollo de este conjunto de criterios, el entorno-portafolio digital del estudiante permite cubrir de modo simultáneo dos propósitos muy claros y aparentemente contradictorios enseñar masivamente, por un lado, y el de enseñar de forma personalizada, por el otro. Sin embargo, en tanto que medio específico debe tener también su propia "gramática", bien en su configuración, en su diseño o modo de empleo por parte de profesores y estudiantes, así como hacerlo contando con los recursos más idóneos para ello y ajustados a sus funcionalidades.

En la tabla 4 siguiente se muestran algunos de los efectos significativos del empleo de una plataforma, organizada o desarrollada de acuerdo con el propósito de calidad y desde la perspectiva de los profesores y 
estudiantes. En síntesis, dichas aportaciones y modalidad de manejo de los diversos recursos, clasificadas según las tres fases de todo aprendizaje, no solo informan, sino que 'educan' también al estudiante en una metodología del proceso de aprendizaje: saber qué hacer, con qué y para qué; hacerlo y tener recursos para analizar o reflexionar, desde su perspectiva, ya sea acerca de lo que se ha hecho, o bien sobre qué se podría mejorar.

\section{Elementos para valorar el enfoque de la experiencia desarrollada}

La reflexión desarrollada acerca del empleo de estos recursos por parte de los diversos profesores y estudiantes, - a partir de una amplia encuesta de más de mil estudiantes de universidades y carreras distintas, que recogía también valoraciones abiertas de los estudiantes (Rué et al. 2010)-, puso de manifiesto la importancia de ciertos rasgos básicos, con respecto a las diversas funcionalidades de dichos entornos. Se validaron tres grandes requerimientos básicos, susceptibles de definir un entorno EPA como tal: que dicho entorno posea unas funcionalidades técnicas básicas y relevantes; que contenga funcionalidades para la interacción (sea esta colaborativa o cooperativa, o tutorial entre iguales o con los profesores); y que incorpore funcionalidades para la reflexión, individual o compartida, con el fin de potenciar la autonomía de los estudiantes mediante la auto-regulación del propio proceso de aprendizaje.

El modelo seguido en el diseño de las

Tabla 4. Efectos formativos del empleo de un EPA que distingue los tres momentos de la acción de aprender

\begin{tabular}{|c|c|c|c|}
\hline AGENTES & $\begin{array}{l}\text { DEFINIR BIEN LA } \\
\text { DOCUMENTACIÓN }\end{array}$ & ESPECIFICAR LA ACCIÓN & $\begin{array}{l}\text { APOYAR, FACILITAR } \\
\text { LA REGULACIÓN }\end{array}$ \\
\hline Profesores & $\begin{array}{c}\text { Incrementa el alcance y la } \\
\text { precisión de la información para } \\
\text { todos los estudiantes. } \\
\text { Ayuda a distinguir la información } \\
\text { básica de la complementaria. } \\
\text { Permite acumular información e ir } \\
\text { seleccionando los mejores } \\
\text { recursos. } \\
\text { Envía el mensaje de que los } \\
\text { contenidos de la materia cobran } \\
\text { sentido en la vida diaria y que el } \\
\text { aprendizaje es más de lo que la } \\
\text { guía o el programa contemplan. }\end{array}$ & $\begin{array}{l}\text { Afina y ajusta la planificación de } \\
\text { las actividades. } \\
\text { Permite ajustar la agenda e } \\
\text { informar anticipadamente de ello, } \\
\text { en función de lo que se considere } \\
\text { oportuno. } \\
\text { Anticipa actividades y da tiempo } \\
\text { para la reflexión. } \\
\text { Permite orientar sobre la reflexión } \\
\text { autónoma de los estudiantes. } \\
\text { Aporta comprensión sobre la } \\
\text { necesidad de explicitar mejor } \\
\text { determinados procesos y el } \\
\text { dominio de ciertas herramientas. }\end{array}$ & $\begin{array}{l}\text { Ayuda a precisar las cualidades } \\
\text { esperadas de los trabajos. } \\
\text { Ayuda a precisar los criterios } \\
\text { previos, a partir de las evidencias } \\
\text { de los estudiantes. } \\
\text { Permite observar los progresos y } \\
\text { entender mejor el proceso } \\
\text { seguido por los estudiantes. } \\
\text { Permite ajustar mejor la carga de } \\
\text { trabajo con respecto a las } \\
\text { producciones esperadas. }\end{array}$ \\
\hline Estudiantes & $\begin{array}{l}\text { Acceso simultáneo a la } \\
\text { información. } \\
\text { Mayor transparencia al facilitar e } \\
\text { incrementar la precisión de la } \\
\text { información recibida. } \\
\text { Actualización de la información } \\
\text { recibida. } \\
\text { Distinción clara entre lo que es } \\
\text { fundamental, lo importante y el } \\
\text { alcance potencial de lo que se } \\
\text { aprende o podría aprender. }\end{array}$ & $\begin{array}{c}\text { Facilita interaccionar, aclarar, } \\
\text { comprender mejor. } \\
\text { Anticipa la reflexión. Incrementa el } \\
\text { interés. } \\
\text { Archivo de procesos de trabajo. } \\
\text { Permite comprender mejor la } \\
\text { importancia de ciertos procesos o } \\
\text { de ciertas herramientas } \\
\text { intelectuales. } \\
\text { Aporta comprensión de lo que } \\
\text { son los procesos de estudio o } \\
\text { sobre su elaboración e } \\
\text { importancia. }\end{array}$ & $\begin{array}{c}\text { Acceso simultáneo a la regulación } \\
\text { Aporta una mejor información } \\
\text { acerca de cómo deben ser las } \\
\text { producciones finales. } \\
\text { Afina el seguimiento y la } \\
\text { evaluación del propio trabajo. } \\
\text { Evidencia cómo el aprendizaje es } \\
\text { un proceso. } \\
\text { Incrementa la transparencia. } \\
\text { Estimula la autocrítica y } \\
\text { la auto-reflexión. }\end{array}$ \\
\hline
\end{tabular}


respectivas EPA y de los recursos empleados por el grupo, trabajando desde diversas plataformas institucionales, reúne las condiciones de diseño que favorecen un "aprendizaje efectivo" (Lombardi, 2007). Esas condiciones son:

- Finalidades y metas transparentes para el trabajo o los aprendizajes.

- Contenidos básicos accesibles o bien orientados, organizados y eventualmente actualizados.

- Diseño de las tareas y de sus objetivos.

- Tareas de alumno: indicadas, renovadas, con una agenda definida.
- Funciones del docente, facilitador, orientador, controlador, evaluador, además de comunicador y tutor.

- Roles de los estudiantes, definidos en la asignación de tareas y en su organización interna para las diversas actividades señaladas.

- Recursos tecnológicos, los empotrados en la plataforma, susceptibles de cubrir todas las funciones requeridas.

- Evaluación diversa, pero considerando el proceso realizado, mediante el testimonio de los portafolios.

Tabla 5. Resultados principales de la encuesta sobre el EPA, sus posibilidades y modo de empleo en los cursos.

\begin{tabular}{|c|c|c|c|}
\hline EJE & ÍTEMS & VALOR & $\mathbf{M}$ \\
\hline \multirow{2}{*}{ Interacción en el aprendizaje } & Trabajar en cooperación & 3,84 & \multirow{2}{*}{3,83} \\
\hline & Compartir trabajo con otros & 3,82 & \\
\hline \multirow{8}{*}{$\begin{array}{l}\text { Facilita el trabajo individual, } \\
\text { en autonomia. }\end{array}$} & Archivo y acceso a los datos & 4,15 & \multirow{8}{*}{3,55} \\
\hline & Trabajo individual & 3,78 & \\
\hline & Sentirse autónomo & 3,61 & \\
\hline & Control agenda de tareas & 3,55 & \\
\hline & Incorporar materiales complementarios & 3,45 & \\
\hline & Visualizar las tareas en su conjunto & 3,44 & \\
\hline & Instrumentos de autoevaluación & 3,28 & \\
\hline & Tener recursos de trabajo & 3,21 & \\
\hline \multirow{6}{*}{ Regulación/co-regulación } & Feed-back profesor & 3,52 & \multirow{6}{*}{3,45} \\
\hline & Identificar habilidades propias/grupo & 3,47 & \\
\hline & Control propia agenda de trabajo & 3,42 & \\
\hline & Control externo & 3,41 & \\
\hline & Facilitar la co-regulación & 3,36 & \\
\hline & Comparar portafolios & 3,23 & \\
\hline \multirow{4}{*}{ Metacognición } & Reflexionar y dejar constancia de ello & 4,00 & \multirow{4}{*}{3,55} \\
\hline & Observar evolución del propio aprendizaje & 3,46 & \\
\hline & Pensar/repensar & 3,39 & \\
\hline & Reflexión proceso & 3,38 & \\
\hline
\end{tabular}

Fuente: elaboración propia, a partir de Rué et al. 2010 
Tal como se esperaba, se observaron diferencias funcionales de empleo de la plataforma por parte de los distintos tipos de estudiantes y profesores, dada la heterogeneidad de cursos, titulaciones, experiencia docente y tipo de estudiantes. No obstante, el análisis ha privilegiado los valores agregados de las valoraciones en su conjunto. Los resultados obtenidos en las valoraciones aportadas por los estudiantes son de dos tipos, cuantitativos y cualitativos. Todos ellos se expresan globalmente, con las medias obtenidas para toda la muestra. Este enfoque ha permitido validar el 'efecto portafolio' en el desarrollo de los cursos y en los aprendizajes, más allá del factor diferencial de cada caso o docente.

Los aspectos más relevantes que arroja el análisis son los siguientes: (a) El grado de dificultad técnica plataforma parece relativamente bajo, de 1,64 (sobre 4 puntos: 1 muy fácil, 4 difícil), con una desviación estándar del 0,737 (valor promedio).

b El grado de valoración de la funcionalidad de la plataforma en relación al archivo de materiales, privacidad y acceso a los datos e informaciones fue de 4.01 (sobre 6 puntos: 1 poco valor, 6 muy valiosa), con una desviación media del 1,47.

Las valoraciones sobre las distintas oportunidades de formación que los estudiantes percibieron mediante el empleo del EPA, en cada uno de los ítems considerados, se reflejan en la tabla siguiente (Tabla 5), ordenada en función de los ejes temáticos que se consideran clave para el aprendizaje. Dichos ejes y sus respectivos valores se han descrito a partir de la clasificación y ordenación de los diversos ítems.

Finalmente, la figura 2 describe las valoraciones agrupadas para cada uno de los ejes descritos. Muestra el alineamiento del conjunto de funciones con las condiciones que exige un aprendizaje de naturaleza profunda, de calidad. Es decir, se verificó que cuando se registraba un adecuado alineamiento entre los entornos para trabajar y las funciones que los concretan se creaban las condiciones básicas que potencian un aprendizaje más profundo, tal como lo han definido Entwistle (1988), Prosser y Trigwell (1999) y Biggs (2003).

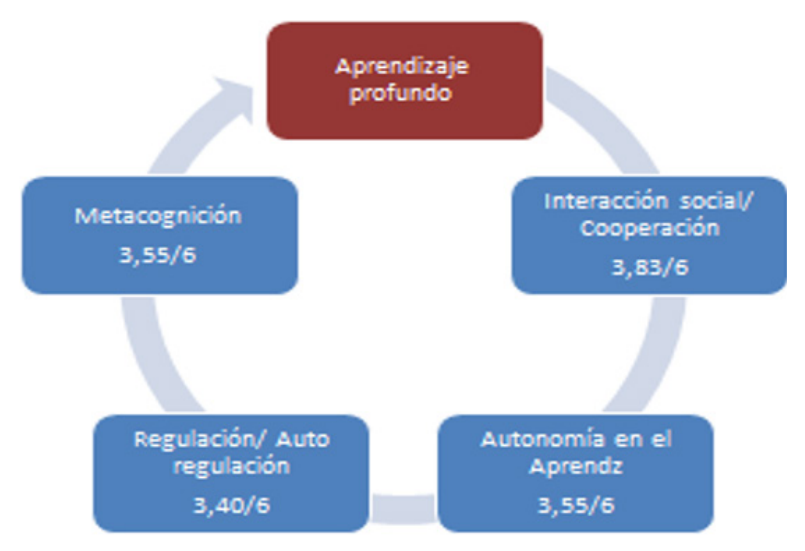

Figura 2. Condiciones necesarias para potenciar un aprendizaje profundo y medias obtenidas

\section{Algunas notas para futuros desarrollos}

La experiencia sintetizada aquí muestra cómo cualquier tentativa del profesorado de desarrollar e implementar en las propias clases un determinado EPA requiere, por su parte, la asunción de un rol que se podría denominar como de 'agente del cambio' en lugar del más habitual 'objeto del cambio', con el que se representa a los usuarios de dichos recursos, consumidores pasivos de plataformas digitales cuyos diseños les son ajenos.

Esta necesidad de 'agencia' del profesor y del liderazgo académico en la introducción y empleo de estos recursos es exigida, entre otros aspectos, por la complejidad de combinar dichos productos, ya que se sitúan en el punto de intersección de la pedagogía, 
la tecnología y el mercado de recursos digitales, ejerciendo estos últimos una importante presión hacia la adquisición y empleo de nuevos y variados dispositivos y herramientas, así como ejercer un efecto negativo, al sustituir la capacidad de agencia profesional de los docentes por unos recursos estandarizados "ready to use", que, precisamente por lo anterior, no serán sometidos a contraste alguno con respecto a su potencial eficacia.

Sin embargo, para que esta capacidad de agencia institucional y docente sea plenamente efectiva, deberá considerarse un apoyo a la formación docente en el empleo de estas plataformas. No olvidemos que hemos sostenido que se trata de "otra forma de aprender" de los estudiantes, y por lo tanto, lo que supone considerar nuevas funcionalidades en la dimensión docente. Es importante que las instituciones velen porque los docentes ejerzan sin problemas añadidos su labor docente, como el de la inseguridad o sentirse aislados o desistidos.

La asunción por parte del docente de aquella actitud de agencia propicia la resolución de la complejidad en el empleo de las tecnologías, lo que se traduce en tres efectos prácticos, vinculados entre sí.

El primero, asumir la propiedad y la responsabilidad del cambio propuesto, de acuerdo con los propios intereses formativos y los de sus estudiantes, mediante la auto-gestión y desarrollo de los propios recursos y de la innovación tecnológica adoptada. Ello presupone también una capacidad docente para personalizar los currículos o las tareas y contenidos específicos en su materia, cuando lo estimen conveniente para favorecer la igualdad de oportunidades en los aprendizajes propuestos.

El segundo, la voluntad de someter la iniciativa desarrollada y las propuestas tecnológicas a los referentes y criterios provenientes de la reflexión pedagógica disponible sobre el conjunto de fenómenos vinculados al aprendizaje y a sus procesos.
Porque las declaraciones o la voluntad institucional de que los profesores sean 'agentes del cambio' no significa que cualquier iniciativa que emprendan vaya a ser relevante, si se ignora el conocimiento público sobre el particular.

El tercero consiste en la necesidad de recoger evidencias que, en la línea de los propósitos formulados, muestren cómo un EPA como el descrito aquí incrementa la colaboración, la regulación, y, en definitiva, la calidad, en los aprendizajes de los estudiantes organizados en grupos medianos y grandes. En otros términos, se trata de que las iniciativas asumidas se sometan a un doble contraste, el del conocimiento público sobre los aspectos vinculados con el aprendizaje y la enseñanza, y el de las evidencias provenientes de las conductas y realizaciones de los estudiantes, precisamente en un contexto particular de grupo o específico de titulación.

A consecuencia de lo anterior, se impone un corolario final. El análisis del funcionamiento de estas plataformas-portafolio hará emerger la necesidad de aportar un apoyo suplementario a determinados grupos de estudiantes con competencias digitales limitadas para que no se queden atrás en su formación. No puede presuponerse que la generalización de estos recursos vaya a darse sin ningún obstáculo, bien sea de competencias o de recursos digitales, por parte de los estudiantes.

\section{Referencias bibliográficas}

Bhattacharya. M, (2001). Electronic Portfolios, Student Reflective Practices, and the Evaluation of Effective Learning'(Minutes of the AAREducation International Education Research Conference, Fremantle, 2-6 December 2001).

Biggs, J. (2003), Teaching for Quality learning at university, Buckinghamshire, 
Society for Research into higher Education and Open University Press.

Brown, M. Dehoney, J. y Millichap, N. (2015). The Next Generation Digital Learning Environment. A Report on Research. ELI Paper, April. 11p. En http://www.educause.edu/research-andpublications [Acceso el 07/05/2015]

Cockburn, T., Carver, T., Shirley, M. y Davies, I., (2007). Using e-portfolio to enable equity students to reflect on and document their skill development, HeinOnline, 15 Waikato L. Rev. 2007. Content downloaded/printed from HeinOnline (http://heinonline.org) August 2009.

EIC, (2018). IV Estudio sobre el uso de la tecnología en la educación. http://www. creadess.org/index.php/informate/desar rollo-humano1/educacion/49424-estudio -sobre-el-uso-de-las-tic-en-la-educacion [Consultado marzo de 2019]

Entwistle, N. (1988). Styles of Learning and Teaching, David Fulton Fiedler Sebastian H.D, Väljataga, T., (2013). Personal learning environments: a conceptual landscape revisited.
eLearning Papers ISSN: 1887-1542, www.openeducationeuropa.eu/en/elear ning_papers n.ํㅜㄴ, November.

Lombardi, M. (2007). Authentic Learning for the 21st Century: An Overview, ELI Paper 1: 2007 ELI Paper 1: May. EDUCAUSE Learning Initiative. May 2007, 16p. https://net.educause.edu/ir/ library/pdf/eli3009.pdf [Acceso el 13/05/ 2015]

Prosser, M. y Trigwell, K. (1999). Understanding Learning and Teaching, on Deep and Surface Learning, Society for Research into Higher Education \& Open University Press, cap.4

Rué, J., Amador, M., Gené, J., Rambla, F.X., Pividori, C., Pividori, I., TorresHostench, O., Bosco, A., Armengol, J., Font, A. (2010), 'Towards an Understanding of Quality in Higher Education: The ELQ/AQA08 Model as a reflection Tool', Quality in Higher Education, 16(3), pp. 285-95.

Rué, J., (2015). Entornos de aprendizaje digitales y calidad de la docencia en Educación Superior. Barcelona, UOC. www.editorialuoc.com 